\title{
Replicated Computations Results (RCR) Report for "Green Simulation: Reusing the Output of Repeated Experiments"
}

\author{
BARRY L. NELSON, Northwestern University
}

\begin{abstract}
"Green Simulation: Reusing the Output of Repeated Experiments" by Feng and Staum describes methods based on likelihood ratio or importance sampling theory for reusing the outputs of simulation experiments at previous parameter settings to augment and improve (by reducing the estimator variance) simulation experiments at new parameter settings. The article presents empirical results for two realistic examples in the area of finance; Matlab code for these examples was made available by the authors. The examples were straightforward to run without extensive knowledge of Matlab, and both experiment and scenario parameters can be altered easily. All experiment results in the article were reproduced.
\end{abstract}

CCS Concepts: • Mathematics of computing $\longrightarrow$ Probability and statistics; Distribution functions;

Additional Key Words and Pharses: Importance sampling, variance reduction, financial engineering

ACM Reference format:

Barry L. Nelson. 2017. Replicated Computations Results (RCR) Report for "Green Simulation: Reusing the Output of Repeated Experiments”. ACM Trans. Model. Comput. Simul. 27, 4, Article 24 (October 2017), 2 pages. https://doi.org/10.1145/3129738

\section{INTRODUCTION}

"Green Simulation: Reusing the Output of Repeated Experiments" by Feng and Staum (2017) describes methods based on likelihood ratio or importance sampling theory for reusing the outputs of simulation experiments at previous parameter settings to augment and improve (by reducing the estimator variance) simulation experiments at new parameter settings. In this context, "parameter settings" refer to the parameters of parametric probability distributions describing the driving random input processes. For instance, if the input process is described by a gamma distribution, then the parameter settings are values for the shape $m$ and scale $\lambda$, which change from experiment to experiment. This environment is relevant, for instance, in the financial industry when computationally intensive simulation for pricing or risk evaluation must take place, say, daily, but each day's simulation differs in the stochastic processes that describe current market conditions. The article establishes asymptotic theory and presents an empirical evaluation.

\section{REPLICATION OF COMPUTATION RESULTS}

Two realistic numerical examples were described in the article-Catastrophe Bond ("CAT bond") Pricing with Compound Losses, and Credit Risk Management-with accompanying Matlab code. No special Matlab Toolboxes are required. The code is executed by running the M-files

Author's address: B. L. Nelson, Department of Industrial Engineering \& Management Sciences, Northwestern University, Evanston, IL 60208-3119, USA.

Permission to make digital or hard copies of all or part of this work for personal or classroom use is granted without fee provided that copies are not made or distributed for profit or commercial advantage and that copies bear this notice and the full citation on the first page. Copyrights for components of this work owned by others than ACM must be honored. Abstracting with credit is permitted. To copy otherwise, or republish, to post on servers or to redistribute to lists, requires prior specific permission and/or a fee. Request permissions from permissions@acm.org.

(C) 2017 ACM 1049-3301/2017/10-ART24 \$15.00

https://doi.org/10.1145/3129738

ACM Transactions on Modeling and Computer Simulation, Vol. 27, No. 4, Article 24. Publication date: October 2017. 
File0_CATBond_main.m and File0_CreditRisk_main.m, respectively. All variables controlling the experiment (e.g., random-number seed) and specifics of the financial problem (e.g., asset valuation horizon) are documented and set at the beginning of each M-file and therefore are easily altered. The default values are the values described in the article.

No difficulties were encountered in running the code, and the results matched those in the article nearly identically, with very small differences easily attributable to Matlab idiosyncrasies. The figures that appear in the article are the primary Matlab output. It is worth noting that these are not computationally trivial examples: on a Dell Latitude E7450 with i7-5600U CPU @ 2.60GHz, the CAT bond example ran overnight, while the Credit Risk example took approximately a day to complete.

\section{ACKNOWLEDGMENTS}

This work is supported by the National Science Foundation under Grant No. CMMI-1634982.

\section{REFERENCE}

Mingbin Feng and Jeremy Staum. 2017. Green simulation: Reusing the output of repeated experiments. Transactions on Modeling and Computer Simulation (2017). To appear.

Received July 2017; accepted August 2017 